4

\title{
Metallization of Textile by Pt Catalyzation in Supercritical Carbon Dioxide and Pt Electroless Plating for Applications in Wearable Devise
}

Mitsuo Sano ${ }^{\mathrm{a}}$, Yuma Tahara ${ }^{\mathrm{b}}$, Tso-Fu Mark Chang ${ }^{\mathrm{a}, \mathrm{c},{ }^{*}}$, Chun-Yi Chen ${ }^{\mathrm{a}, \mathrm{c}}$, Tomoko Hashimoto $^{\mathrm{b}}$, Hiromichi Kurosu ${ }^{\mathrm{b}}$, Masato Sone $\mathrm{e}^{\mathrm{a}, \mathrm{c}}$

${ }^{a}$ Precision and Intelligence Laboratory, Tokyo Institute of Technology, 4259 Nagatsuta,

Midori-ku, Yokohama 226-8503, Japan

${ }^{\mathrm{b}}$ School of Natural Science and Ecological Awareness, Nara Women's University, Kita-Uoya Nishimachi, Nara-shi, Nara 630-8506, Japan

c CREST, Japan Science and Technology Agency, 4259 Nagatsuta, Midori-ku, Yokohama 226-8503, Japan

*Corresponding Author. Tel.: +81-45-924-5631

E-mail address: chang.m.aa@m.titech.ac.jp (Mark Chang) 


\section{Abstract}

2 In this study, an electroless plating method involved Pt catalyzation in supercritical 3 carbon dioxide $\left(\mathrm{sc}-\mathrm{CO}_{2}\right)$ and conventional $\mathrm{Pt}$ electroless plating was used for the 4 metallization of polyamide 6,6 (PA66) textiles. The catalyzation was conducted either 5 by conventional method at atmospheric pressure or in $\mathrm{sc}-\mathrm{CO}_{2}$ with bis 6 (2,4-pentandionato)-platinum. After the catalyzation process, the conventional $\mathrm{Pt}$ 7 electroless plating method was performed for the Pt catalyzed PA66 textiles. Surface of 8 the Pt coated PA66 was observed by an optical microscope and a scanning electron 9 microscope. The Pt coating by the conventional catalyzation had a rough surface, and 10 the surface did not show the textile outline because of the dissolution of the textile in 11 the conventional catalyzation solution. The textile metallized by the sc- $\mathrm{CO}_{2}$ catalyzation 12 showed a metallic bright coating, and the Pt coating was observed on the surface of each 13 fibers. These results indicate that the $\mathrm{sc}-\mathrm{CO}_{2}$ catalyzation is a promising method to 14 catalyze the PA66 textile. 


\section{Introduction}

2 Wearable device is an advanced technology that involves metallization of textiles for

3 applications in various fields [1-3]. One of the challenging points in fabrication of the

4 wearable device is the deposition of metallic materials on the textile. There are many

5 methods that could be applied to deposit metallic materials on the textile, such as

6 sputtering [4], chemical vapor deposition [5], laser-assisted metallization [6] and electrostatic

7 spray deposition [7]. Among the metallization methods, electroless plating is the most

8 promising method in the industrial level because of its simple operation and low cost

9 process. Therefore, studies on fabrication of the fiber coated with metallic materials by

10 electroless deposition have attracted much attention. However, there are still some

11 points needed to be improved in the conventional electroless plating method. First, a

12 pretreatment process is usually needed to improve adherence of the metallic coatings on

13 the fibers, and toxic chemicals are often used in this step. In this case, residual of the

14 toxic substances raises concerns for practical use of the fibers in clothing. Second, the

15 fibers can be damaged or even lost because of the strong acid used in the pretreatment.

16 Supercritical carbon dioxide $\left(\mathrm{sc}-\mathrm{CO}_{2}\right)$ is non-polar and has physical properties

17 between those of gas and liquid. Most importantly, $\mathrm{CO}_{2}$ is non-toxic and abundant in 
1 the environment. Hence, $\mathrm{sc}-\mathrm{CO}_{2}$ is used as non-toxic solvents in extractions, separations,

2 chemical reactions, and various other applications [8]. Recently, several studies on the

3 deposition using $\mathrm{sc}-\mathrm{CO}_{2}$ have been reported, such as a supercritical fluid deposition

4 [9-13] and copper electroplating with $\mathrm{sc}-\mathrm{CO}_{2}$ using organo copper complex as the

5 source of copper [12]. Sc- $\mathrm{CO}_{2}$ is reported to be able to penetrate into the structure of

6 polymers because of the non-polar and zero surface tension properties. Based on this,

7 metallization of polymeric substrate by electroless plating utilizing $\mathrm{sc}-\mathrm{CO}_{2}$ has been

8 demonstrated [13]. The $\mathrm{sc}-\mathrm{CO}_{2}$ is used in the catalyzation step with an organo $\mathrm{Pd}$

9 complex as the source of the Pd catalyst, and coverage and adhesion of the metal

10 coatings on the polymer substrate, such as Kapton® polyimide film, are reported to be

11 improved. Therefore, we suggest the $\mathrm{sc}-\mathrm{CO}_{2}$ catalyzation (SCC) can also be applied in

12 metallization of polyamide 6,6 (PA66) textile to solve the problems of the toxic

13 chemicals used in the pretreatment and to improve adhesion of the metal coating on the

14 textile. PA66 is also known as nylon 6,6, which is extensively used in the fiber industry

15 and various other fields because of the high crystalline melting point, good resistance to

16 hydrocarbons, high strength, high biocompatibility, and simple fabrication process [14].

17 Thus, PA66 textile is selected as the substrate material in this study,

18 The biocompatibility is the most important factor for the applications in wearable 
1 devices. Therefore, Pt was chosen as the catalyst in the electroless plating and the metal

2 deposited on the textile in this study. This is because, Pt has better biocompatibility than

3 Pd often used in the conventional catalyzation [15].

4 In this paper, metallization of the PA66 textiles with an improved biocompatibility by

5 having Pt as the catalyst and the metallic coating on the PA66 textile is reported. Also,

6 the SCC would be applied to eliminate the toxic chemicals often used in the

7 catalyzation step and improve the quality of the Pt coatings on the PA66 textile. The

8 PA66 metallization method proposed in this study is promising for applications of the

9 metallized fibers in the wearable devices because the concerns from the residual toxic

10 substances and the possible metal allergy symptom caused by Pd and Ni could all be

11 eliminated.

\section{Experimental}

\subsection{Experimental apparatus}

The high pressure apparatus (Japan Spectra Company) used in this investigation is shown in Fig. 1. The reaction chamber was made of stainless steel 316 vessel with an

17 inner volume of $50 \mathrm{ml}$. The reaction chamber was placed in a temperature-controlled air 18 bath to control the experimental temperature. The PA66 textile was positioned in the reaction chamber using a plastic-coated platinum wire. 
1 2.2. Materials and sample preparation

2 In this study, $\mathrm{CO}_{2}$ having purity of $99.99 \%$ was used. $\mathrm{A} \mathrm{PdCl}_{2} / \mathrm{SnCl}_{2}$ solution 3 consisted of $\mathrm{PdCl}_{2}(100 \mathrm{mg} / \mathrm{l}), \mathrm{SnCl}_{2}(10 \mathrm{mg} / \mathrm{l})$ and $\mathrm{HCl}(87.5 \mathrm{~g} / \mathrm{l})$ was used for the 4 conventional catalyzation (Conv-Cat). Bis (2,4-pentandionato)-platinum was used as the 5 source of $\mathrm{Pt}$ in the SCC. The Pt plating bath was commercially available electrolyte 6 purchased from MATEX Japan, and it was consisted of MATEX PLATINUM basic 7 bath $(500 \mathrm{ml} / 1 \mathrm{~L})$, MATEX PLATINUM reduction agent $(10 \mathrm{ml} / 1 \mathrm{~L}), \mathrm{NH}_{3}$ solution $8(50 \mathrm{ml} / \mathrm{1L})$, and ion-exchanged water $(460 \mathrm{ml} / 1 \mathrm{~L})$. Platinum concentration in the 9 plating bath was $2 \mathrm{~g} / \mathrm{L}$. The $\mathrm{pH}$ of this bath was 12 . The textile used in this study was made from PA66 fibers. Image of the untreated PA66 is shown in Fig. 2(a).

\subsection{Catalyzation process}

No pretreatment was conducted in this study. Two types of catalyzation procedures were performed. The temperature used in the Conv-Cat was room temperature. The time used in the Conv-Cat was $10 \mathrm{~s}$. The temperature of the SCC was $353 \mathrm{~K}$, and the pressure for the SCC was $15 \mathrm{MPa}$. The time of the SCC was $2 \mathrm{~h}$.

After the catalyzation process, the conventional electroless plating (Conv-EP) was performed. The catalyzed textile was immersed in the Pt electroless plating bath at 333 
Surfaces morphology of the Pt coated PA66 textiles was observed by an optical microscope (OM, KEYENCE: VHV-5000) and a scanning electron microscope (SEM,

4 JEOL: JSM-7500).

5

\section{3. Results and discussion}

$7 \quad 3.1$ Surface morphology

8 Images of the PA66 textiles treated by the Conv-Cat and 3.5 and $4 \mathrm{~h}$ of the 9 Conv-EP are shown in Fig. 2(b) and (c), respectively. By comparing appearance of the Conv-Cat and Conv-EP treated PA66 textiles with the untreated PA66 textile shown in 11 Fig. 2(a), surface of the Conv-Cat treated PA66 fibers was rough and a few white particulates was observed under the OM. The results were suggested to be caused by the acid used in the Conv-Cat step. Rough surface is usually beneficial in metallization of polymers. However, surface of the Conv-Cat treated PA66 fibers did not show obvious appearance of any metallic coating after $3.5 \mathrm{~h}$ of the Conv-EP process. When the $\mathrm{Pt}$ plating time was increased to $4 \mathrm{~h}$, the appearance was still similar to that of the $3.5 \mathrm{~h}$ case.

The SCC and Conv-EP treated PA66 textiles are shown in Fig. 3. After the Conv-EP for 3.5 h, shown in Fig. 3(a), surface of the PA66 fibers was found to be covered by bright $\mathrm{Pt}$ coating. The Pt coatings seemed to be very thin in the $\mathrm{OM}$ observation. Therefore, a longer plating time of $4 \mathrm{~h}$ was used to increase the coating 
thickness. As shown in Fig. 3(b), much thicker Pt coating was observed on the PA66

2 fibers. Although the Pt coating became much thicker after $4 \mathrm{~h}$ of the plating, the outline

3

4 of the fibers could still be seen.

More details of the Pt coatings could be observed from the SEM images in Fig. 4. For, the Conv-Cat and Conv-EP treated PA66 textiles, the surfaces were rough, and it was difficult to identify outline of the fibers because boundary between each fibers was not clear, shown in Fig. 4(a) and (b). Also, there was not much difference when the Conv-EP was increased from 3.5 to $4 \mathrm{~h}$. These results were in agreement with the surface morphology observed by the OM and confirmed that the Conv-Cat process was not appropriate for metallization of the PA66 textiles. This is because; damage of the PA66 and deformation of the fibers occurred in the Conv-Cat step. The acid used in the $\mathrm{PdCl}_{2} / \mathrm{SnCl}_{2}$ solution can react with the PA66 polymer chains. Then, subsequent disruption of the hydrogen bonds between the polymer chains occurred [16].

In Fig. 4(c), the SEM image shows that the SCC and Conv-EP for $3.5 \mathrm{~h}$ could produce pinhole-free and smooth Pt coatings on the PA66 fibers. Furthermore, the Pt coating on the surface of each fiber still showed outline of the fibers. No peeled-off part was observed. These results demonstrate that the SCC was an excellent catalyzation process to improve the adherence of the metal coatings on the textile. The adherence was improved by the anchoring effect, which metal roots were formed under surface of the polymers to anchor the metal coatings $[11,13]$. The metal roots were formed by impregnation of the Pt catalysts into the polymer by the sc- $\mathrm{CO}_{2}$. The solubility of bis (2,4-pentandionato)-platinum in pure sc- $\mathrm{CO}_{2}$ is high, and $\mathrm{sc}-\mathrm{CO}_{2}$ can penetrate into the structures of polymers because of the non-polar and zero surface tension properties [17]. 
1 So, the catalyst could be transferred into the polymer structure to form catalyst seeds in

2 the polymer. Then, reduction of the metal ions could be initiated at the catalyst seeds for

3 form metal roots to anchor the metal coatings.

4 Fig. 4(d) shows the Pt coating by the SCC and Conv-EP of $4 \mathrm{~h}$. With a longer 5 plating time, the Pt coating thickness is expected to be higher. The result showed that 6 the outline of the fibers was still obvious with a thicker Pt coating, but some cracks

7 were observed. The cracks were suggested to be mostly caused by the differences in 8 elastic constant between Pt and PA66. A longer plating time could also lead to a larger 9 internal stress in the plated film, which can lead to the cracks or peel-offs.

\section{Conclusions}

In this study, Pt metallization of the PA66 textiles was realized by the sc- $\mathrm{CO}_{2}$ catalyzation and the conventional electroless plating. When the PA66 textiles were catalyzed in the $\mathrm{PdCl}_{2} / \mathrm{SnCl}_{2}$ mixture solution, it was difficult to have complete coverage of the Pt coatings on the PA66 fibers, and the fibers were found to be damaged. The damage was suggested to be caused by the acid used in the Conv-Cat process. On the other hand, complete coverage of the PA66 textiles with defect-free bright Pt coatings was obtained when the SCC was applied. For the Pt metallization of PA66 utilizing the SCC, improved bio-compatibility by replacing Pd with Pt, exclusion of the toxic chemicals used in the catalyzation step, and Pt coatings with enhanced adherence on the PA66 are the promising points for the applications in wearable devices. 


\section{Acknowledgments}

3 This work has been supported by The Grant-in-Aid for Scientific Research (B)

4 (JSPSKAKENHIGrantNumber26282013) and CREST Project operated by the Japan

$5 \quad$ Science and Technology Agency (JST).

6

7

8

9

10

11

12

13

14

15

16

17

18 


\section{Reference}

3 [1] J. Brand, M. Kok, M. Koetse, M. Cauwe, R. Verplancke, F. Bossuyt, M. Jablonski, J.

4 Vanfleteren, Flexible and stretchable electronics for wearable health devices,

5 Solid-State Electron. 113 (2015) 116-120.

6 [2] A. Affanni, G. Chiorboli, Design and characterization of a real-time, wearable,

7 endosomatic electrodermal system, Meas. 75 (2015) 111-121.

8 [3] G.K. Lewis Jr, M. D. Langer, C.R. Henderson Jr, R. Ortiz, Design and evaluation of

9 a wearable self-applied therapeutic ultrasound device for chronic myofascial pain,

$10 \quad$ Ultrasound Med. Biol. 39 (2013) 1429-1439.

11 [4] P. Sigmund, Mechanisms and theory of physical sputtering by particle impact, Nucl.

12 Instrum. Meth. 27 (1987) 1-20.

13 [5] K.L. Choy, Chemical vapour deposition of coatings, Prog. Mater Sci. 48 (2003)

$14 \quad 57-170$.

15 [6] P. Rytlewski, Laser-assisted metallization of composite coatings containing copper(II)

16 acetylacetonate and copper(II) oxide or copper(II) hydroxide, Surf. Coat. Technol. 259 (2014)

$17 \quad 660-666$. 
1 [7] M. Barletta, A. Gisario, V. Tagliaferri, Electrostatic spray deposition (ESD) of polymeric

2 powders on thermoplastic (PA66) substrate, Surf. Coat. Technol. 201 (2006) 296-308

3 [8] T. Clifford, Fundamentals of supercritical fluids, Oxford University Press, United

4 Kingdom, (1999).

5 [9] D.P. Long, J.M. Blackburn, J.J. Watkins, Chemical Fluid Deposition: A hybrid

6 technique for low-temperature metallization, Adv.Mater.12 (2000) 913-915.

7 [10] S.T. Chung, Y.C. Chuang, S.Y. Chiu, W.T. Tsai, Effect of $\mathrm{H}_{3} \mathrm{PO}_{3}$ concentration on

8 the electrodeposition of nanocrystalline $\mathrm{Ni}-\mathrm{P}$ deposited in an emulsified supercritical

$9 \quad \mathrm{CO}_{2}$ bath, Electrochim. Acta 58 (2011) 571- 577.

10 [11 H. Adachi, K. Taki, S. Nagamine, A. Yusa, M. Ohshima, Supercritical carbon

11 dioxide assisted electroless plating on thermoplastic polymers, J. Supercrit. Fluids 49

12 (2009) 265-270.

13 [12] T. Shimizu, Y. Ishimoto, T.F.M. Chang, H. Kinashi,T. Nagoshi, T. Sato, M. Sone,

$14 \mathrm{Cu}$ wiring into nano-scale holes by electrodeposition in supercritical carbon dioxide

15 emulsified electrolyte with a continuous-flow reaction system, J. Supercrit. Fluids 90

16 (2014) 60-64.

17 [13] B.H. Woo, M. Sone, A. Shibata, C. Ishiyama, S. Edo, M. Tokita, J. Watanabe, Y.

18 Higo, Impregnation of $\mathrm{Ni}-\mathrm{P}$ metal into polymer substrate via catalyzation in $\mathrm{sc}-\mathrm{CO}_{2}$ and 19 electroless plating in sc-CO2 emulsion, Surf. Coat. Technol. 204 (2010) 1785-1792. 
1 [14] H. Qin, Q. Su, S. Zhang, B. Zhao, M. Yang, Thermal stability and flammability of

2 polyamide 66/montmorillonite nanocomposites, Polym. 44 (2003) 7533-7538.

3 [15] J.C. Wataha, C.T. Hanks, Biological effects of palladium and risk of using

4 palladium in dental casting alloys, J. Oral Rehabil. 23 (1996) 309-320.

5 [16] R.M. Mcdonogh, C.J.D. Fell, A.G. Fane, Characteristics of membranes formed by

6 acid dissolution of polyamides, J. Membr. Sci. 31 (1987) 321-336.

7 [17] S. Yoda, A. Hasegawa, H. Suda, Y. Uchimaru, K. Haraya, T. Tsuji, K. Otake,

8 Preparation of a platinum and palladium/polyimide nanocomposite film as a precursor

9 of metal-doped carbon molecular sieve membrane via supercritical impregnation, Chem.

10 Mater. 16 (2004) 2363-2368.

11

12

13

14

15

16

17

18

19 
$3 \quad$ Figure captions

4 Figure 1. (a) $\mathrm{CO}_{2}$ gas tank, (b) $\mathrm{CO}_{2}$ liquidization unit, (c) liquidization unit, (d) high

5 pressure pump, (e) thermal bath, (f) reaction-cell (PEEK-lined SUS316L), (g) substrate,

6 (h) cross stirrer, (i) back pressure regulator, and (j) trap.

7

8 Figure 2, OM images of the (a) untreated PA66 textile and PA66 textiles treated by (b)

9 Conv-Cat and Conv-EP for $3.5 \mathrm{~h}$ and (c) Conv-Cat and Conv-EP for $4 \mathrm{~h}$.

11 Figure 3, OM images of the PA66 textiles treated by (a) SCC and Conv-EP for $3.5 \mathrm{~h}$ 12 and (b) SCC and Conv-EP for $4 \mathrm{~h}$.

14 Figure 4, SEM images of surface of the Pt coatings by (a) Conv-Cat and Conv-EP for $153.5 \mathrm{~h}$, (b) Conv-Cat and Conv-EP for $4 \mathrm{~h}$, (c) SCC and Conv-EP for $3.5 \mathrm{~h}$, (d) SCC and 16 Conv-EP for $4 \mathrm{~h}$. 


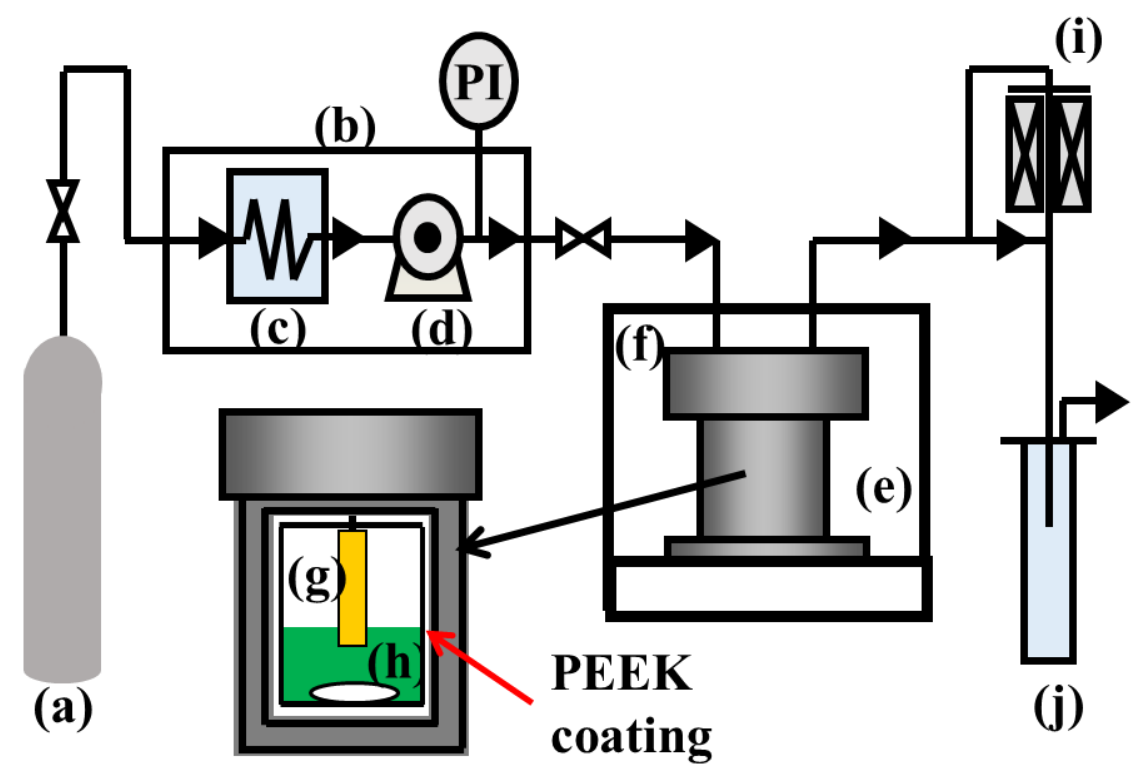

Figure 1. (a) $\mathrm{CO}_{2}$ gas tank, (b) $\mathrm{CO}_{2}$ liquidization unit, (c) liquidization unit, (d) high pressure pump, (e) thermal bath, (f) reaction-cell (PEEK-lined SUS316L), (g) substrate, (h) cross stirrer, (i) back pressure regulator, and (j) trap. 


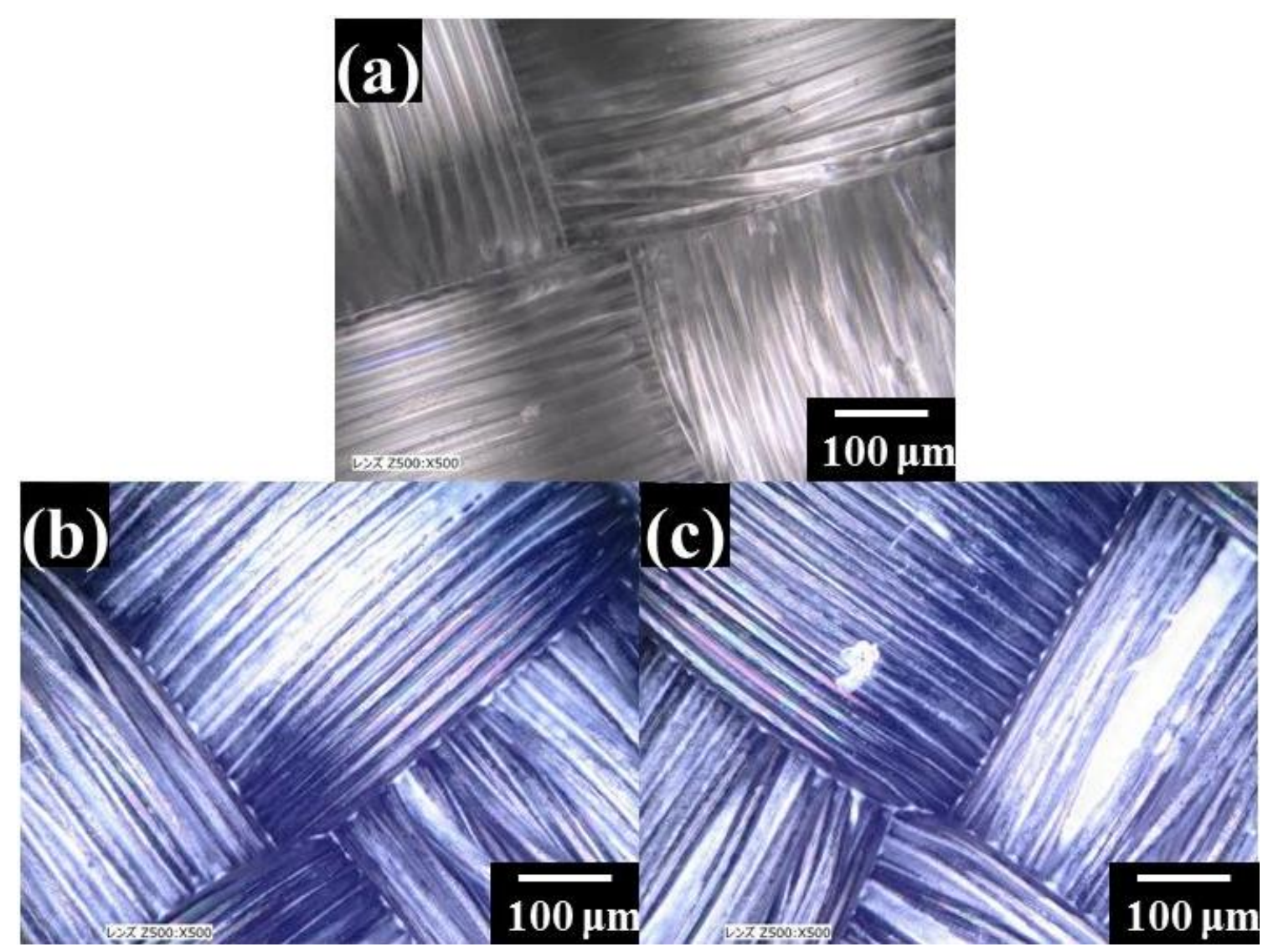

Figure 2, OM images of the (a) untreated PA66 textile and PA66 textiles treated by (b) Conv-Cat and Conv-EP for $3.5 \mathrm{~h}$ and (c) Conv-Cat and Conv-EP for $4 \mathrm{~h}$. 


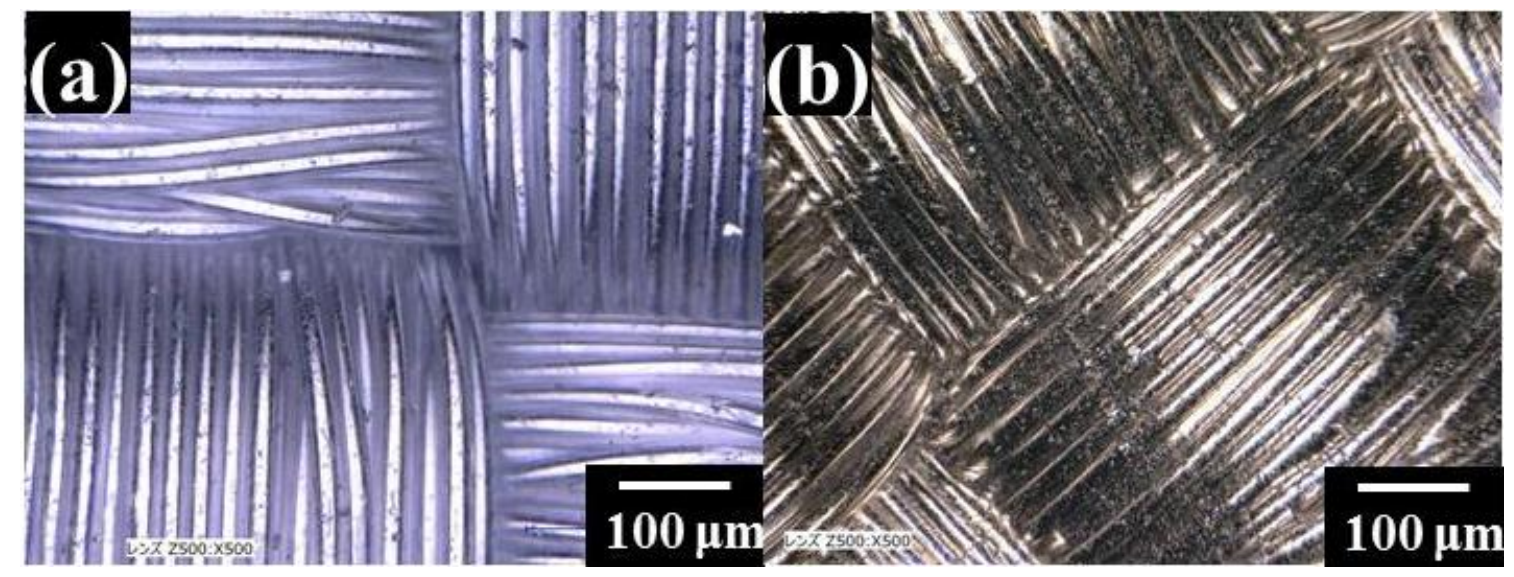

Figure 3, OM images of the PA66 textiles treated by (a) SCC and Conv-EP for $3.5 \mathrm{~h}$ and (b) SCC and Conv-EP for $4 \mathrm{~h}$. 


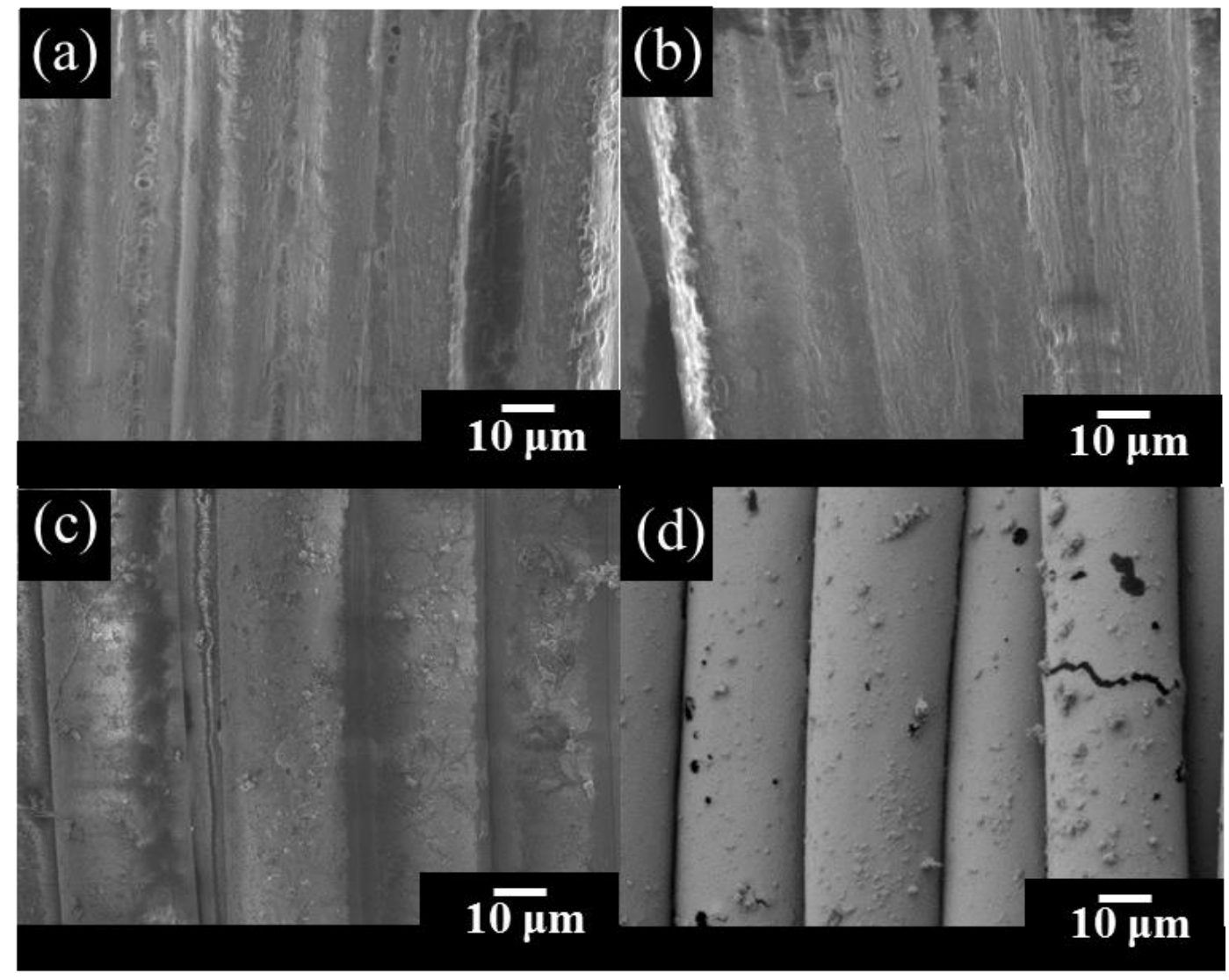

Figure 4, SEM images of surface of the Pt coatings by (a) Conv-Cat and Conv-EP for $3.5 \mathrm{~h}$, (b) Conv-Cat and Conv-EP for $4 \mathrm{~h}$, (c) SCC and Conv-EP for $3.5 \mathrm{~h}$, (d) SCC and Conv-EP for $4 \mathrm{~h}$. 


\section{Pt coated PA66 textiels}

\section{$\underline{\text { Supercritical CO}} \mathbf{C O}_{2}$ Catalyzation (SCC)}
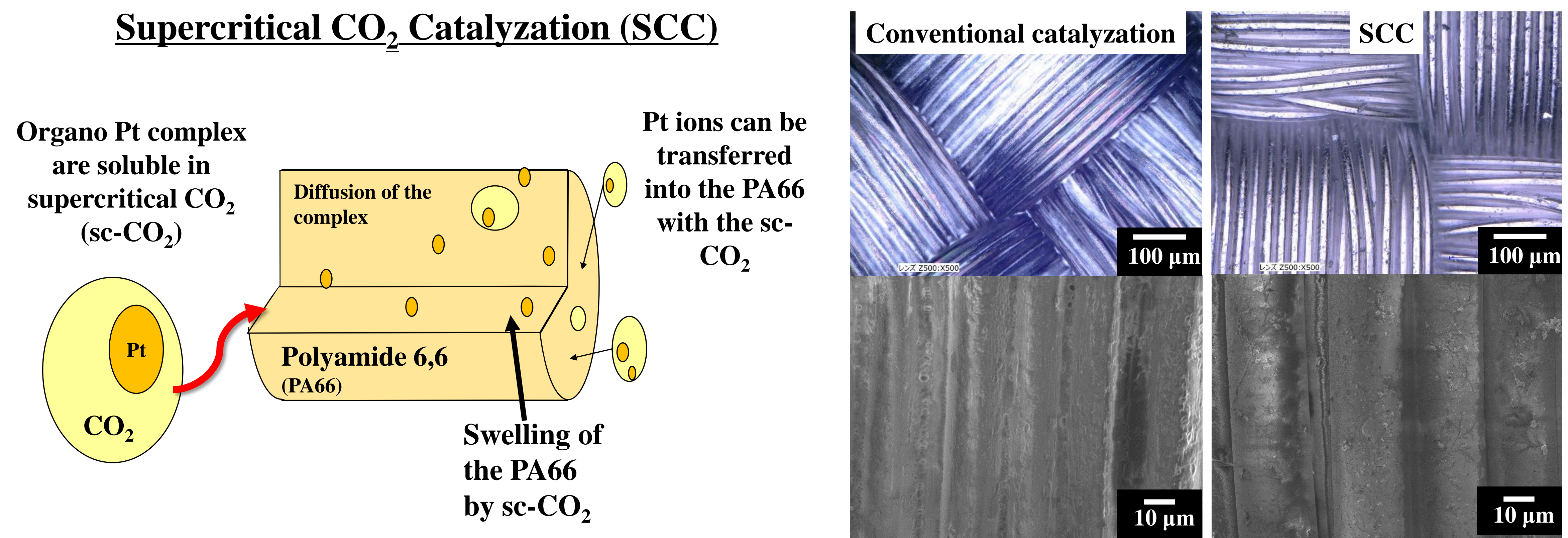

Not coated with Pt

$\underline{\text { Uniform Pt coating }}$ 